\title{
Effect of seed treatments and storage containers on seed quality parameters of popular rice (Oryza sativa L.) cultivar
}

\author{
A.Harsha ${ }^{1}$, B. Rajeswari ${ }^{2}$, D. Krishnaveni ${ }^{3}$, K. Keshavulu ${ }^{4}$ \\ ${ }^{1}$ Department of Plant Pathology, College of Agriculture, Rajendranagar, Hyderabad. \\ ${ }^{2}$ Professor, Dept. of Plant Pathology, College of Agriculture, Rajendranagar, Hyderabad \\ ${ }^{3}$ Principal Scientist (Plant Pathology),IIRR, Rajendranagar, Hyderabad. \\ ${ }^{4}$ Director, Telangana state seed certification agency, Hyderabad. \\ *Email: rao.harsha97@gmail.com
}

\begin{abstract}
Rice cv. RNR 15048 (Telangana sona) was treated with three fungicides viz., thiram @ $0.3 \%$, carbendazim@0.2\% and mancozeb@0.25\% and three biocontrol agents viz., T. harzianum, T. viride and $P$. flourescence and stored in gunny bag and polythene bag was assessed for seed quality parameters $v i z$, seed germination and seedling vigour at biomonthly intervals. Seeds treated with thiram $\left(\mathrm{T}_{1}\right)$ enhanced seed germination and vigour as compared with untreated seeds (control) in polythene bag and gunny bag storage. Seeds treated with thiram and stored in polythene bag increased seed germination $(89.5 \%)$ and seedling vigour (SVI-I, 1696) (SVI-II, 1039) at the end of eight months storage period as against the seeds stored in gunny bag which recorded less seed germination $(87.2 \%)$ and seedling vigour (SVI-I, 1650) (SVI-II, 924) respectively. The other seed treatments viz., carbendazim, $P$. fluorescence, $T$. harzianum and $T$. viride were also found effective in increasing germination and seedling vigour as compared with untreated seeds in polythene bag storage.
\end{abstract}

Keywords: Seed treatment, seed germination, polythene bag, gunny bag, RNR 15048 .

Paper cited: Harsha, A., Rajeswari, B., Krishnaveni, D. and Keshavulu, K. (2017). Effect of seed treatments and storage containers on seed quality parameters of popular rice (Oryza sativa L.) cultivar. South Asian Journal of Food Technology and Environment, 3(1):502-506.

\section{Introduction}

Rice is the main staple food for more than half of the world's population. Seeds lose their viability and vigour due to changes in environmental conditions like temperature and humidity (Roberts, 1972), storing of paddy seeds in polythene bag maintained viability and vigour. Seed treatment is an efficient technology replacing wasteful foliar and soil application of chemicals. In recent years, use of Trichoderma spp. as a bioagent was found effective in controlling diseases (Cook and Baker, 1983). Seed treatment should be done as a routine practice as it is a cheap insurance against possible disasters at a later stage. Thrimurty (1986) has reported the efficacy of carbendazim (Bavistin) in reducing disease severity.

\section{Materials and Methods}

Rice cv. RNR 15048 (fine grain rice variety) was inoculated with Drechslera oryzae (a) $10^{9}$ conidia $/ \mathrm{ml}$ and shade dried for $24 \mathrm{~h}$. The seeds were treated with fungicides and biocontrol agents as dry seed treatment along with untreated (control). After seed treatment the seeds were equally divided into two parts and filled in two containers viz., gunny bag $(25 \times 15$ $\mathrm{cm})$ and polythene bag $\left(\begin{array}{lll}10 & \mathrm{x} & 12 \mathrm{~cm}\end{array}\right)$ commercially available 700 guage. The seeds 
thus packed were stored under ambient laboratory conditions of Indian Institute of Rice Research, Rajendranagar, Hyderabad for a period of eight months from August 2015 to April 2016. The laboratory experiments were conducted in four replications adopting Factorial experiment laid out in CRD taking seed treatment as one factor and container as another Germination (\%): The germination per cent was assessed by adopting rolled paper towel method as prescribed by International Seed Testing Association Rules (ISTA, 1996). Germination paper towels of standard size (440 $\times 330$ ) were dipped in sterile distilled water and drained. Hundred seeds of each rice seed sample were distributed at equidistance. Another towel, similarly was laid over and labelled. The towels were then rolled, drained of excess water, placed in aerated polythene bag and incubated in an upright position at $28 \pm 2^{\circ} \mathrm{C}$ in a BOD incubator. Four hundred seeds with hundred seeds of each replication were examined. On fourteenth day, germination percentage was recorded.

$$
\begin{aligned}
& \text { Germination }(\%) \\
& =\frac{\text { Number of normal seedlings }}{\text { Total number of seeds }} \times 100
\end{aligned}
$$

Seedling vigour index: Ten normal seedlings from each replication of germination test were taken separately for measuring seedling length in centimeters $(\mathrm{cm})$. The seedling length comprises of root length and shoot length. The same seedlings which were taken for measuring seedling length were oven dried in an hot air oven at $60^{\circ} \mathrm{C}$ for $24 \mathrm{~h}$ for obtaining seedling dry weight and expressed in milligrams (mg). Seedling vigour index I and II were calculated by using the formula given by Abdul Baki and Anderson (1973).

SVI I $=$ Seedling length $(\mathrm{cm}) \times$ Germination percentage $(\%)$

SVI II = Seedling dry weight $(\mathrm{mg}) \times$ Germination percentage $(\%)$ factor (Table 1). At biomonthly intervals, seed samples were drawn from different seed treatments and containers and tested for seed health parameters. The data pertaining to percentage values were analyzed after converting them into transformed values (Gomez and Gomez, 1984).

\section{Results and Discussion}

Effect of seed treatments and storage containers on germination and seedling vigour of rice cv. RNR15048: Results of Table 2 indicates that irrespective of the seed treatments and containers, the germination percentage was gradually decreased with increase in period of storage. The per cent germination was above Minimum Seed Certification Standards $(80 \%)$ at six months storage period. However, the interaction between seed treatments and storage containers were found non-significant at eight months period of storage. Seeds stored in polythene bag (700 gauge) was recorded higher seed germination than gunny bag during all the periods of seed storage. The decline in seed germination over initial storage period was high in gunny bag (13.2\%) as compared to polythene bag storage $(11.7 \%)$. Seed treatments gave significant impact by improving the seed germination over untreated control. Among the seed treatments, thiram $\left(\mathrm{T}_{1}\right)(88.3 \%)$ followed by carbendazim $\left(\mathrm{T}_{2}\right)(87.8 \%)$ recorded higher seed germination over other seed treatments during all the storage periods. Treated seed stored in polythene bag was found effective in maintaining the germination over untreated seeds stored in gunny bag. Untreated seed stored in gunny bag showed minimum germination (77.7 \%). Seeds treated with $P$. fluorescence $(86.1 \%)$, mancozeb $\left(\mathrm{T}_{3}\right)(84.2 \%)$, $T$. viride $\left(\mathrm{T}_{4}\right)$ $(81.2 \%)$ and $T$. harzianum $\left(\mathrm{T}_{6}\right) \quad(79.0 \%)$ recorded higher seed germination over untreated control $\left(\mathrm{T}_{7}\right)(78.2 \%)$. The results are in agreement with the reports of Imolehin (1983) 
and, Zulkifi et al., (1991) who reported reduction in seed germination due to $D$. oryzae. The results of the present study also indicated that seed treatment with thiram provided greater protection to rice seeds and improved viability which is in agreement with Yogalakshmi et al., (1996).

Seedling vigour index was decreased gradually with the period of seed storage in all the seed treatments and storage containers (Table 3 \& 4). Seeds stored in polythene bag showed higher seedling vigour index than gunny bag in all the seed treatments especially in thiram $\left(\mathrm{T}_{1}\right)$ during all the storage periods irrespective of the containers. Irrespective of the seed treatments, seeds stored in polythene bag had significantly higher seedling vigour index over gunny bag. At the end of eight months period of seed storage, seeds treated with thiram and stored in polythene bag recorded maximum SVI - I \& II $(1039,981)$, while the untreated seeds stored in gunny bag had minimum SVI - I \& II (1118, 702). Germination percentage, seedling vigour and storability were found high in thiram $\left(\mathrm{T}_{1}\right)$ followed by carbendazim $\left(\mathrm{T}_{2}\right), P$. fluorescence $\left(\mathrm{T}_{5}\right)$, mancozeb $\left(\mathrm{T}_{3}\right)$, T. viride $\left(\mathrm{T}_{4}\right)$ and $T$. harzianum $\left(\mathrm{T}_{6}\right)$ as compared to untreated rice seeds. The decrease in seed vigour index might be attributed to age induced decline in germination, decrease in root and shoot length. The enhancement in germination and seed vigour observed in fungicide treated seeds are in agreement with the results of the studies undertaken by Misra and Dharamvir (1991) and Sachan and Agarwal (1994).

Table 1: Details of seed treatments and storage containers adopted in the experiment

\begin{tabular}{|l|l|}
\hline Factor $1:$ seed treatments & Factor 2: storage container \\
\hline T1 : Thiram @ $3 \mathrm{~g} / \mathrm{kg}$ & $\mathrm{C} 1:$ Gunny bag \\
\hline T2 : Carbendazim @ 2g/kg & $\mathrm{C} 2:$ Polythene bag \\
\hline T3: Mancozeb @ $2.5 \mathrm{~g} / \mathrm{kg}$ & \\
\hline T4: T. viride @ $10 \mathrm{~g} / \mathrm{kg}$ & \\
\hline T5 : P. flourescence @ $10 \mathrm{~g} / \mathrm{kg}$ & \\
\hline T6 : T. harzianum @ $2.5 \mathrm{~g} / \mathrm{kg}$ & \\
\hline T7 : Untreated seeds ( control) & \\
\hline
\end{tabular}

Table 2: Effect of seed treatments and storage containers on germination (\%) of rice cv. RNR 15048

\begin{tabular}{|c|c|c|c|c|c|c|c|c|c|c|c|c|c|}
\hline \multirow{2}{*}{$\begin{array}{l}\text { Treat- } \\
\text { ment }\end{array}$} & \multirow{2}{*}{$\begin{array}{l}\text { Before } \\
\text { storage }\end{array}$} & \multicolumn{3}{|c|}{$2 \mathrm{MAS}$} & \multicolumn{3}{|c|}{ 4MAS } & \multicolumn{3}{|c|}{$6 \mathrm{MAS}$} & \multicolumn{3}{|c|}{$8 \mathrm{MAS}$} \\
\hline & & $\mathrm{C}_{1}$ & $\mathrm{C}_{2}$ & Mean & $\mathrm{C}_{1}$ & $\mathrm{C}_{2}$ & Mean & $\mathrm{C}_{1}$ & $\mathrm{C}_{2}$ & Mean & $\mathrm{C}_{1}$ & $\mathrm{C}_{2}$ & Mean \\
\hline $\mathrm{T}_{1}$ & $\begin{array}{l}98.2 \\
(82.6)^{*}\end{array}$ & $\begin{array}{l}93.7 \\
(75.5)\end{array}$ & $\begin{array}{l}95.2 \\
(77.4)\end{array}$ & $\begin{array}{l}94.5 \\
(76.2)\end{array}$ & $\begin{array}{l}92.5 \\
(74.1)\end{array}$ & $\begin{array}{l}93.5 \\
(75.2)\end{array}$ & $\begin{array}{l}93.0 \\
(74.6)\end{array}$ & $\begin{array}{l}89.7 \\
(71.3)\end{array}$ & $\begin{array}{l}91.5 \\
(73.0)\end{array}$ & $\begin{array}{l}90.6 \\
(72.1)\end{array}$ & $\begin{array}{l}87.2 \\
(69.0)\end{array}$ & $\begin{array}{l}89.5 \\
(71.0)\end{array}$ & $\begin{array}{l}88.3 \\
(70.0)\end{array}$ \\
\hline $\mathrm{T}_{2}$ & $\begin{array}{l}98.2 \\
(82.6)\end{array}$ & $\begin{array}{l}93.0 \\
(74.6)\end{array}$ & $\begin{array}{l}93.5 \\
(75.2)\end{array}$ & $\begin{array}{l}93.2 \\
(74.9)\end{array}$ & $\begin{array}{l}91.2 \\
(72.8)\end{array}$ & $\begin{array}{l}91.7 \\
(73.3)\end{array}$ & $\begin{array}{l}91.5 \\
(73.0)\end{array}$ & $\begin{array}{l}89.5 \\
(71.0)\end{array}$ & $\begin{array}{l}89.7 \\
(71.3)\end{array}$ & $\begin{array}{l}89.6 \\
(71.2)\end{array}$ & $\begin{array}{l}87.0 \\
(68.8)\end{array}$ & $\begin{array}{l}88.7 \\
(70.4)\end{array}$ & $\begin{array}{l}87.8 \\
(69.6)\end{array}$ \\
\hline $\mathrm{T}_{3}$ & $\begin{array}{l}95.7 \\
(78.1) \\
\end{array}$ & $\begin{array}{l}90.0 \\
(71.5) \\
\end{array}$ & $\begin{array}{l}90.7 \\
(72.3) \\
\end{array}$ & $\begin{array}{l}90.6 \\
(72.2) \\
\end{array}$ & $\begin{array}{l}87.7 \\
(69.5) \\
\end{array}$ & $\begin{array}{l}90.2 \\
(71.8) \\
\end{array}$ & $\begin{array}{l}89.0 \\
(70.6) \\
\end{array}$ & $\begin{array}{l}84.7 \\
(67.0) \\
\end{array}$ & $\begin{array}{l}87.2 \\
(69.1) \\
\end{array}$ & $\begin{array}{l}86.0 \\
(68.0) \\
\end{array}$ & $\begin{array}{l}83.2 \\
(65.8) \\
\end{array}$ & $\begin{array}{l}85.2 \\
(67.4) \\
\end{array}$ & $\begin{array}{l}84.2 \\
(66.6) \\
\end{array}$ \\
\hline $\mathrm{T}_{4}$ & $\begin{array}{l}95.2 \\
(77.4)\end{array}$ & $\begin{array}{l}90.7 \\
(72.3)\end{array}$ & $\begin{array}{l}90.5 \\
(72.0)\end{array}$ & $\begin{array}{l}90.3 \\
(71.9)\end{array}$ & $\begin{array}{l}85.0 \\
(67.2)\end{array}$ & $\begin{array}{l}89.2 \\
(70.8)\end{array}$ & $\begin{array}{l}87.1 \\
(69.0)\end{array}$ & $\begin{array}{l}83.5 \\
(66.0)\end{array}$ & $\begin{array}{l}86.7 \\
(68.6)\end{array}$ & $\begin{array}{l}85.1 \\
(67.3)\end{array}$ & $\begin{array}{l}80.0 \\
(63.4)\end{array}$ & $\begin{array}{l}82.5 \\
(65.2)\end{array}$ & $\begin{array}{l}81.2 \\
(64.3)\end{array}$ \\
\hline $\mathrm{T}_{5}$ & $\begin{array}{l}96.0 \\
(78.5)\end{array}$ & $\begin{array}{l}92.5 \\
(74.1)\end{array}$ & $\begin{array}{l}92.7 \\
(74.4)\end{array}$ & $\begin{array}{l}92.6 \\
(74.2)\end{array}$ & $\begin{array}{l}91.2 \\
(72.8)\end{array}$ & $\begin{array}{l}90.5 \\
(72.0)\end{array}$ & $\begin{array}{l}90.8 \\
(72.4)\end{array}$ & $\begin{array}{l}88.7 \\
(70.4)\end{array}$ & $\begin{array}{l}89.2 \\
(70.8)\end{array}$ & $\begin{array}{l}89.0 \\
(70.6)\end{array}$ & $\begin{array}{l}86.0 \\
(68.0)\end{array}$ & $\begin{array}{l}86.2 \\
(68.2)\end{array}$ & $\begin{array}{l}86.1 \\
(68.1)\end{array}$ \\
\hline $\mathrm{T}_{6}$ & $\begin{array}{l}95.0 \\
(77.1)\end{array}$ & $\begin{array}{l}88.2 \\
(69.9)\end{array}$ & $\begin{array}{l}91.7 \\
(73.3) \\
\end{array}$ & $\begin{array}{l}90.0 \\
(71.6) \\
\end{array}$ & $\begin{array}{l}85.5 \\
(67.6) \\
\end{array}$ & $\begin{array}{l}87.0 \\
(68.8) \\
\end{array}$ & $\begin{array}{l}86.2 \\
(68.2) \\
\end{array}$ & $\begin{array}{l}81.2 \\
(64.3) \\
\end{array}$ & $\begin{array}{l}80.5 \\
(63.7) \\
\end{array}$ & $\begin{array}{l}80.8 \\
(64.0) \\
\end{array}$ & $\begin{array}{l}78.7 \\
(62.5) \\
\end{array}$ & $\begin{array}{l}79.2 \\
(62.9) \\
\end{array}$ & $\begin{array}{l}79.0 \\
(62.7) \\
\end{array}$ \\
\hline $\mathrm{T}_{7}$ & $\begin{array}{l}95.0 \\
(77.1)\end{array}$ & $\begin{array}{l}89.2 \\
(70.8)\end{array}$ & $\begin{array}{l}90.7 \\
(72.3) \\
\end{array}$ & $\begin{array}{l}90.0 \\
(71.6)\end{array}$ & $\begin{array}{l}84.0 \\
(66.4)\end{array}$ & $\begin{array}{l}88.0 \\
(69.7) \\
\end{array}$ & $\begin{array}{l}86.0 \\
(68.0)\end{array}$ & $\begin{array}{l}80.7 \\
(63.9)\end{array}$ & $\begin{array}{l}80.0 \\
(63.4)\end{array}$ & $\begin{array}{l}80.3 \\
(63.7)\end{array}$ & $\begin{array}{l}77.7 \\
(61.8) \\
\end{array}$ & $\begin{array}{l}78.7 \\
(62.5)\end{array}$ & $\begin{array}{l}78.2 \\
(62.2)\end{array}$ \\
\hline Mean & $\begin{array}{l}96.0 \\
(78.0)\end{array}$ & $\begin{array}{l}91.0 \\
(72.7) \\
\end{array}$ & $\begin{array}{l}92.1 \\
(73.8) \\
\end{array}$ & & $\begin{array}{l}88.1 \\
(70.0)\end{array}$ & $\begin{array}{l}90.0 \\
(71.7) \\
\end{array}$ & & $\begin{array}{l}85.4 \\
(67.7)\end{array}$ & $\begin{array}{l}86.4 \\
(68.6)\end{array}$ & & $\begin{array}{l}82.8 \\
(65.6)\end{array}$ & $\begin{array}{l}84.3 \\
(66.8)\end{array}$ & \\
\hline
\end{tabular}


A. Harsha, B. Rajeswari, D. Krishnaveni, K. Keshavulu

\begin{tabular}{|c|c|c|c|c|c|c|c|c|c|c|c|c|}
\hline & \multicolumn{3}{|c|}{$2 \mathrm{MAS}$} & \multicolumn{3}{|c|}{ 4MAS } & \multicolumn{3}{|c|}{ 6MAS } & \multicolumn{3}{|c|}{ 8MAS } \\
\hline & $\mathrm{T}$ & $\mathrm{C}$ & $\mathrm{T} \times \mathrm{C}$ & $\mathrm{T}$ & $\mathrm{C}$ & $T \times C$ & $\mathrm{~T}$ & $\mathrm{C}$ & $T \times C$ & $\mathrm{~T}$ & $\mathrm{C}$ & $T \times C$ \\
\hline SEm \pm & 0.39 & 0.21 & 0.56 & 0.34 & 0.18 & 0.49 & 0.33 & 0.17 & 0.46 & 0.34 & \begin{tabular}{|l|}
0.18 \\
\end{tabular} & 0.49 \\
\hline CD at $5 \%$ & 1.14 & 0.61 & 1.61 & 0.99 & 0.52 & 1.40 & 0.94 & 0.50 & 1.33 & 0.99 & 0.53 & NS \\
\hline
\end{tabular}

Each value is a mean of four replications

$$
\begin{array}{ll}
\mathrm{C}_{1} \text { - Gunny bag } & \mathrm{T}_{1} \text { - Thiram @ 3g/kg } \\
\mathrm{C}_{2} \text { - Polythene bag } & \mathrm{T}_{2} \text { - Carbendazim @2g/kg } \\
& \mathrm{T}_{3} \text { - Mancozeb@2.5g/kg } \\
& \mathrm{T}_{4} \text { - Trichoderma viride@10g/kg } \\
& \mathrm{T}_{5} \text { - Pseudomonas fluorescence@ } 10 \mathrm{~g} / \mathrm{kg} \\
& \mathrm{T}_{6} \text { - Trichoderma harzianum @ } 10 \mathrm{~g} / \mathrm{kg} \\
& \mathrm{T}_{7} \text { - Untreated seeds }
\end{array}
$$

\begin{tabular}{|c|c|c|c|c|c|c|c|c|c|c|c|c|c|}
\hline \multirow{2}{*}{$\begin{array}{l}\text { Treat } \\
\text { ment }\end{array}$} & \multirow{2}{*}{$\begin{array}{l}\text { Before } \\
\text { storage }\end{array}$} & \multicolumn{3}{|c|}{ 2MAS } & \multicolumn{3}{|c|}{ 4MAS } & \multicolumn{3}{|c|}{ 6MAS } & \multicolumn{3}{|c|}{ 8MAS } \\
\hline & & $\mathrm{C}_{1}$ & $\mathrm{C}_{2}$ & Mean & $\overline{C_{1}}$ & $\mathrm{C}_{2}$ & Mean & $\mathrm{C}_{1}$ & $\mathrm{C}_{2}$ & Mean & $\overline{C_{1}}$ & $\mathrm{C}_{2}$ & Mean \\
\hline $\mathrm{T}_{1}$ & 2500 & 2172 & 2370 & 2271 & 1931 & 2240 & 2085 & 1759 & 1773 & 1766 & 1650 & 1696 & 1673 \\
\hline $\mathrm{T}_{2}$ & 2474 & 1920 & 2320 & 2120 & 1820 & 2213 & 2016 & 1536 & 1890 & 1713 & 1400 & 1782 & 1591 \\
\hline $\mathrm{T}_{3}$ & 2379 & 2144 & 1995 & 2069 & 1816 & 2089 & 1952 & 1352 & 1778 & 1565 & 1253 & 1674 & 1463 \\
\hline $\mathrm{T}_{4}$ & 2352 & 1762 & 2153 & 1957 & 1623 & 2082 & 1853 & 1357 & 1542 & 1450 & 1250 & 1329 & 1289 \\
\hline $\mathrm{T}_{5}$ & 2420 & 1995 & 2222 & 2108 & 1765 & 2163 & 1964 & 1480 & 1776 & 1628 & 1448 & 1563 & 1505 \\
\hline $\mathrm{T}_{6}$ & 2296 & 1838 & 2054 & 1946 & 1597 & 1894 & 1745 & 1299 & 1492 & 1396 & 1192 & 1324 & 1258 \\
\hline $\mathrm{T}_{7}$ & 2230 & 1704 & 2139 & 1921 & 1535 & 1993 & 1764 & 1278 & 1492 & 1385 & 1118 & 1188 & 1153 \\
\hline \multirow[t]{3}{*}{ Mean } & 2379 & 2567 & 2179 & & 1727 & 2096 & & 1438 & 1678 & & 1330 & 2092 & \\
\hline & & \multicolumn{3}{|c|}{$2 \mathrm{MAS}$} & \multicolumn{3}{|c|}{ 4MAS } & \multicolumn{3}{|c|}{ 6MAS } & \multicolumn{3}{|c|}{ 8MAS } \\
\hline & & $\mathrm{T}$ & $\mathrm{C}$ & $\mathrm{T} \times \mathrm{C}$ & $\mathrm{T}$ & $\mathrm{C}$ & $T \times C$ & $\mathrm{~T}$ & $\mathrm{C}$ & $\mathrm{T} \times \mathrm{C}$ & $\mathrm{T}$ & $\mathrm{C}$ & $\mathrm{T} \times \mathrm{C}$ \\
\hline & $\mathbf{m} \pm$ & 25. & 13.8 & 36.7 & 30.9 & 16.5 & 43.7 & 27.1 & 14.4 & 38.3 & 18.1 & 9.70 & 25.6 \\
\hline & $D$ at $5 \%$ & 74. & \begin{tabular}{l|l|}
6 & 39.64 \\
\end{tabular} & 104.88 & 88.31 & 47.20 & NS & 77.36 & 41.35 & 109.40 & 51.80 & 27.69 & 73.26 \\
\hline
\end{tabular}

\begin{tabular}{|c|c|c|c|c|c|c|c|c|c|c|c|c|c|}
\hline \multirow{2}{*}{$\begin{array}{l}\text { Treat } \\
\text { ment }\end{array}$} & \multirow{2}{*}{$\begin{array}{l}\text { Before } \\
\text { storage }\end{array}$} & \multicolumn{3}{|c|}{$2 \mathrm{MAS}$} & \multicolumn{3}{|c|}{ 4MAS } & \multicolumn{3}{|c|}{ 6MAS } & \multicolumn{3}{|c|}{$8 \mathrm{MAS}$} \\
\hline & & $\mathrm{C}_{1}$ & $\mathrm{C}_{2}$ & Mean & $\mathrm{C}_{1}$ & $\mathrm{C}_{2}$ & Mean & $\mathrm{C}_{1}$ & $\mathrm{C}_{2}$ & Mean & $\mathrm{C}_{1}$ & $\mathrm{C}_{2}$ & Mean \\
\hline $\mathrm{T}_{1}$ & 2029 & 1843 & 1864 & 1853 & 1639 & 1721 & 1680 & 1221 & 1439 & 1330 & 924 & 1039 & 981 \\
\hline $\mathrm{T}_{2}$ & 1920 & 1666 & 1721 & 1693 & 1499 & 1676 & 1588 & 1091 & 1283 & 1187 & 840 & 1032 & 936 \\
\hline $\mathrm{T}_{3}$ & 1861 & 1576 & 1643 & 1609 & 1508 & 1511 & 1509 & 1026 & 1205 & 1116 & 763 & 867 & 815 \\
\hline $\mathrm{T}_{4}$ & 1801 & 1557 & 1660 & 1608 & 1394 & 1490 & 1442 & 1045 & 1183 & 1114 & 798 & 814 & 806 \\
\hline $\mathrm{T}_{5}$ & 1920 & 1583 & 1794 & 1689 & 1466 & 1598 & 1532 & 1080 & 1246 & 1163 & 887 & 827 & 857 \\
\hline $\mathrm{T}_{6}$ & 1750 & 1506 & 1611 & 1558 & 1352 & 1462 & 1407 & 992 & 1117 & 1054 & 702 & 702 & 702 \\
\hline $\mathrm{T}_{7}$ & 1684 & 1438 & 1524 & 1481 & 1229 & 1377 & 1303 & 890 & 931 & 911 & 766 & 613 & 690 \\
\hline \multirow[t]{3}{*}{ Mean } & 1852 & 1595 & 1688 & & 1441 & 1548 & & 1049 & 1201 & & 811 & 842 & \\
\hline & & \multicolumn{3}{|c|}{$2 \mathrm{MAS}$} & \multicolumn{3}{|c|}{ 4MAS } & \multicolumn{3}{|c|}{ 6MAS } & \multicolumn{3}{|c|}{$8 \mathrm{MAS}$} \\
\hline & & $\mathrm{T}$ & $\mathrm{C}$ & $\mathrm{T} \times \mathrm{C}$ & $\mathrm{T}$ & $\mathrm{C}$ & $\mathrm{T} \times \mathrm{C}$ & $\mathrm{T}$ & C & $\mathrm{T} \times \mathrm{C}$ & $\mathrm{T}$ & $\mathrm{C}$ & $\mathrm{T} \times \mathrm{C}$ \\
\hline \multicolumn{2}{|c|}{ SEm \pm} & 23. & 12.6 & 33.5 & 20.0 & 10.7 & 28.3 & 19.0 & 10.1 & 26.9 & 38.6 & 20.6 & 54.6 \\
\hline \multicolumn{2}{|c|}{ CD at $5 \%$} & 67. & 36.2 & NS & 57.2 & 30.5 & NS & 54.4 & 29.0 & NS & 110.2 & NS & NS \\
\hline
\end{tabular}

Table 3: Effect of seed treatments and storage containers on seedling vigour index - I of rice cv. RNR 15048

Each value is a mean of four replications

Table 4: Effect of seed treatments and storage containers on seedling vigour index - II of rice cv. RNR 15048

Each value is a mean of four replications 


\section{References}

1. Abdul Baki, A.A. and Anderson, J.D. (1973). Vigour determination in soybean seed by multiple criteria. Crop Science. 13: 630-633.

2. Cook, R.J. and Baker, K.F. (1983). The nature and practice of biological control of plant pathogens. American Phytopathological Society St.Paul Minnesota, Academic Press, pp.539.

3. Gomez, K.A. and Gomez, A.A. (1984). Statistical procedures for agricultural research. $2^{\text {nd }}$ edition. John Wiley and Sons Inc. New York, USA. 13- 175.

4. Imolehin, E.D.( 1983). Rice seed borne fungi and their effect on seed germination. Plant Disease. 67: 1334513.

5. ISTA (1996). International Rules Seed Testing Association. Seed Science \& Technology, 13: 299-513.

6. Misra, A.K. and Dharavir (1991). Efficacy of fungicides - XLVI: Effect of fungicidal seed treatment against heavy inoculum pressure of certain fungi causing discolouration of paddy seeds. Indian Phytopathology. 43: 175-178.

7. Roberts, E.H. (1972). Cytological, genetical and metabolic changes associated with loss of viability. In Viability of Seeds. Academic Press Ltd, New York. pp 253306.

8. Sachan, I.P. and Agarwal, V.K. (1994). Efficacy of seed treatment of discoloured seeds of rice on seed borne inoculum, germination and seedling vigour. Seed Research. 22(1): 45-49.

9. Thrimurthy, V.S. (1986). Studies on chemical control of sheath rot of rice. Pesticides. 9: 20-21.

10. Yogalakshmi, J., Ponnusamy, A.S. and Karivaratharaju, T.V. (1996). Seed storage potential of rice hybrid CORH -
I and parental lines. Madras Agriculture Journal. 83(11): 729-732.

11. Zulkifi, E.J. and Castano, J. (1991). Effect of grain discolouration in upland rice on some yield components. International Rice Research Notes. 16: 20.

\begin{tabular}{|ll|}
\hline Received & : May, 2017 \\
Revised & : May, 2017 \\
Published & : June, 2017 \\
\hline
\end{tabular}

